\title{
Spontaneous Tumor Lysis Syndrome in a Patient with Metastatic Small Cell Lung Cancer: A Case Report
}

\author{
Boonphiphop Boonpheng Ghulam Murtaza David Ginn \\ Department of Internal Medicine, East Tennessee State University, Johnson City, TN, USA
}

\section{Keywords}

Tumor lysis syndrome $\cdot$ Spontaneous tumor lysis syndrome $\cdot$ Small cell lung cancer

\begin{abstract}
Tumor lysis syndrome is an oncologic emergency that usually occurs after chemotherapy in patients with hematologic malignancies. Tumor lysis syndrome is rare in cases of solid tumors, especially when it occurs spontaneously. Herein, we present a case of spontaneous tumor lysis syndrome in a 55-year-old woman who presented with dyspnea and was found to have extensive metastatic small cell lung cancer. She developed acute oliguric renal failure and multiple electrolyte abnormalities requiring hemodialysis. The findings of this case suggest that clinicians should maintain a high index of suspicion for patients with malignancies who demonstrate the classic symptom of laboratory abnormalities even in the absence of chemotherapy.

(C) 2017 The Author(s)

Published by S. Karger AG, Basel
\end{abstract}

\section{Introduction}

Tumor lysis syndrome (TLS) is an oncologic emergency that frequently occurs in patients with a rapidly proliferating malignancy, commonly hematologic, after the initiation of cytotoxic therapy [1]. TLS is characterized by a massive release of intracellular potassium, phosphate, and nucleic acid metabolites into the systemic circulation, which can result in cardiac arrhythmia, seizure, or acute renal failure, all of which could be life-threatening. 


\section{Case Reports in Oncology}

Boonpheng et al.: Spontaneous Tumor Lysis Syndrome in a Patient with Metastatic Small Cell Lung Cancer: A Case Report

Prompt recognition and intervention is therefore important to prevent further complications [2]. However, spontaneous lysis of solid tumors is considered rare. Herein, we present a case of spontaneous TLS in a 55-year-old woman that occurred shortly after she was diagnosed with metastatic small cell lung cancer.

\section{Case Presentation}

A 55-year-old female smoker with a history of chronic obstructive pulmonary disease and diabetes presented to the emergency department with worsening dyspnea and respiratory distress. Her vital signs were significant for tachypnea and tachycardia. A physical examination revealed wheezing and decreased breath sounds in the left upper lung field. Chest radiography showed left upper lung airspace disease. The initial presentation prompted the diagnosis of pneumonia with acute respiratory failure. She was admitted to the intensive care unit and started receiving intravenous antibiotics, fluid resuscitation, and noninvasive ventilatory support. Owing to right upper quadrant pain and a high alkaline phosphate level, abdominal ultrasonography was conducted, which showed an enlarged liver with multiple masses. Once the patient's condition stabilized, chest and abdomen computed tomography examinations were performed, which showed a $7-\mathrm{cm}$ mass in the left upper lung lobe associated with obstructive pneumonitis involving much of the left upper lobe (Fig. 1) as well as extensive bilateral mediastinal and left hilar, axillary, and supraclavicular lymphadenopathy and multiple liver metastases. Histopathologic examination of a liver biopsy specimen indicated high-grade small cell neuroendocrine cancer. Magnetic resonance imaging of the brain yielded normal findings. Finally, the diagnosis was established as extensive stage small cell lung cancer.

On hospital day 4, however, oliguria was detected. Serum biochemistry tests showed elevated creatinine and potassium levels, and therefore, sepsis-associated acute kidney injury was initially considered. Subsequent laboratory examinations revealed increasing levels of potassium (maximum $5.6 \mathrm{mEq} / \mathrm{L}$ ), phosphorus (maximum $8.4 \mathrm{mg} / \mathrm{dL}$ ), and uric acid (maximum $11.3 \mathrm{mg} / \mathrm{dL}$ ). Accordingly, spontaneous TLS was diagnosed on the basis of the characteristic symptom of multiple electrolyte-related abnormalities. Aggressive treatment in the form of fluid resuscitation, phosphate binders, allopurinol, and rasburicase was initiated. Nonetheless, her renal function continued to deteriorate, with the creatinine level increasing from $0.5 \mathrm{mg} / \mathrm{dL}$ upon admission to $7.9 \mathrm{mg} / \mathrm{dL}$ over 6 days. The departments of oncology and nephrology were consulted, and the consensus was to conduct daily hemolysis after the first cycle of palliative chemotherapy comprising cisplatin and etoposide. No major complications were reported thereafter. However, her renal recovery was poor and she became dialysis dependent.

\section{Discussion}

The patient in this case demonstrated spontaneous TLS caused by small cell lung cancer, which is a highly proliferative solid tumor. The patient had oliguric renal failure and characteristic laboratory abnormalities including hyperkalemia, hyperuricemia, and hyperphosphatemia in the setting of widespread metastatic disease. To the best of our knowledge, only 4 case reports of spontaneous TLS associated with small cell lung cancer have been reported in the literature [3-6]. 


\section{Case Reports in Oncology}

Boonpheng et al.: Spontaneous Tumor Lysis Syndrome in a Patient with Metastatic Small Cell Lung Cancer: A Case Report

Typically, TLS results from the massive release of intracellular electrolytes and metabolites when large numbers of tumor cells are lysed by cytotoxic chemotherapy or, rarely, spontaneously. Most commonly, TLS occurs in cases of rapidly growing malignancies with a high tumor burden, especially hematologic malignancies such as Burkitt lymphoma or acute lymphoblastic leukemia [1]. However, cases of TLS involving solid tumors have been reported in the literature $[7,8]$. The classic findings of TLS are hyperkalemia, hyperphosphatemia with secondary hypocalcemia, and hyperuricemia. These electrolyte abnormalities can lead to cardiac arrhythmia, seizure, or acute renal failure, all of which could be life-threatening without prompt recognition and treatment.

The risk factors for TLS include bulky tumors, extensive metastatic diseases including visceral or bone marrow involvement, elevated serum uric acid or lactate dehydrogenase levels, high white blood cell count $(>50,000$ cells $/ \mu \mathrm{L}$ ), tumors with a high sensitivity to antineoplastic treatment, and underlying renal dysfunction [7]. TLS involving solid tumors is assumed to share similar risk factors $[8,9]$, although its spontaneous occurrence in tumor types with relatively low response rates to cytotoxic therapy or without the classic rapid growth kinetics [7], such as that observed in hepatocellular [10], pancreatic [11], ovarian cancer [12], and malignant melanoma [13], has been reported. In our patient, the risk factors included extensive metastatic disease and small cell lung cancer, a rapidly proliferative tumor cell type.

Although rare, TLS involving solid tumors is associated with a higher mortality rate than that reported for hematologic malignancies [7]. Current guidelines emphasize both preventive measures and monitoring for high-risk patients undergoing chemotherapy [1, 2]. Aggressive fluid resuscitation to maintain a large urine output remains the mainstay of management. Allopurinol, a xanthine oxidase inhibitor, or rasburicase can be used to lower the serum uric acid level. However, the utility of urine alkalization remains controversial. Hemodialysis is an option for patients who develop severe renal failure or refractory electrolyte abnormalities, as in our patient.

In conclusion, spontaneous TLS should be considered in the differential diagnosis for patients who demonstrate the classic electrolyte abnormalities or renal failure in the setting of malignancy even without cytotoxic treatment, as observed in our case. Prophylactic treatment could be a suitable option for patients with multiple risk factors for spontaneous TLS.

\section{Acknowledgment}

We would like to thank Editage (www.editage.com) for English language editing.

\section{Statement of Ethics}

The authors declare that there is no ethical conflict to disclose.

\section{Disclosure Statement}

The authors declare that there is no conflict of interest regarding the publication of this article. 


\section{Case Reports in Oncology}

\begin{tabular}{l|l}
\hline Case Rep Oncol 2017;10:392-395 \\
\hline DOI: 10.1159/000474937 & $\begin{array}{l}\text { C 2017 The Author(s). Published by S. Karger AG, Basel } \\
\text { www.karger.com/cro }\end{array}$ \\
\hline
\end{tabular}

Boonpheng et al.: Spontaneous Tumor Lysis Syndrome in a Patient with Metastatic Small Cell Lung Cancer: A Case Report

\section{References}

$\checkmark 1$ Cairo MS, Coiffier B, Reiter A, Younes A; TLS Expert Panel: Recommendations for the evaluation of risk and prophylaxis of tumour lysis syndrome (TLS) in adults and children with malignant diseases: an expert TLS panel consensus. Br J Haematol 2010;149:578-586.

-2 Coiffier B, Altman A, Pui CH, Younes A, Cairo MS: Guidelines for the management of pediatric and adult tumor lysis syndrome: an evidence-based review. J Clin Oncol 2008;26:2767-2778

3 Jallad B, Hamdi T, Latta S, Alhosaini MN, Kheir F, Iroegbu N: Tumor lysis syndrome in small cell lung cancer: a case report and review of the literature. Onkologie 2011;34:129-131.

-4 Padhi P, Singh S: Spontaneous tumor lysis syndrome in a patient with metastatic small cell carcinoma of the lung. J Cancer Sci Ther 2012;4:164-166.

-5 Weerasinghe C, Zaarour M, Arnaout S, Garcia G, Dhar M: Spontaneous tumor lysis syndrome in small cell lung cancer: a rare complication. World J Oncol 2015;6:464-471.

-6 Kanchustambham V, Saladi S, Patolia S, Stoeckel D: Spontaneous tumor lysis syndrome in small cell lung cancer. Cureus 2017;9:e1017.

7 Gemici C: Tumour lysis syndrome in solid tumours. Clin Oncol (R Coll Radiol) 2006;18:773-780

-8 Mirrakhimov AE, Ali AM, Khan M, Barbaryan A: Tumor lysis syndrome in solid tumors: an up to date review of the literature. Rare Tumors 2014;6:5389.

9 Baeksgaard L, Sorensen JB: Acute tumor lysis syndrome in solid tumors - a case report and review of the literature. Cancer Chemother Pharmacol 2003;51:187-192.

10 Mehrzad R, Saito H, Krahn Z, Feinstein A: Spontaneous tumor lysis syndrome in a patient with metastatic hepatocellular carcinoma. Med Princ Pract 2014;23:574-576.

11 Saleh RR, Rodrigues J, Lee TC: A tumour lysis syndrome in a chemotherapy naive patient with metastatic pancreatic adenocarcinoma. BMJ Case Rep 2015;2015:bcr2014207748.

-12 Okamoto K, Kinoshita T, Shimizu M, Okura I, Kawada A, Mizobuchi K, et al: A case of spontaneous tumor lysis syndrome in a patient with ovarian cancer. Case Rep Obstet Gynecol 2015;2015:461870.

13 Mouallem M, Zemer-Wassercug N, Kugler E, Sahar N, Shapira-Frommer R, Schiby G: Tumor lysis syndrome and malignant melanoma. Med Oncol 2013;30:364.

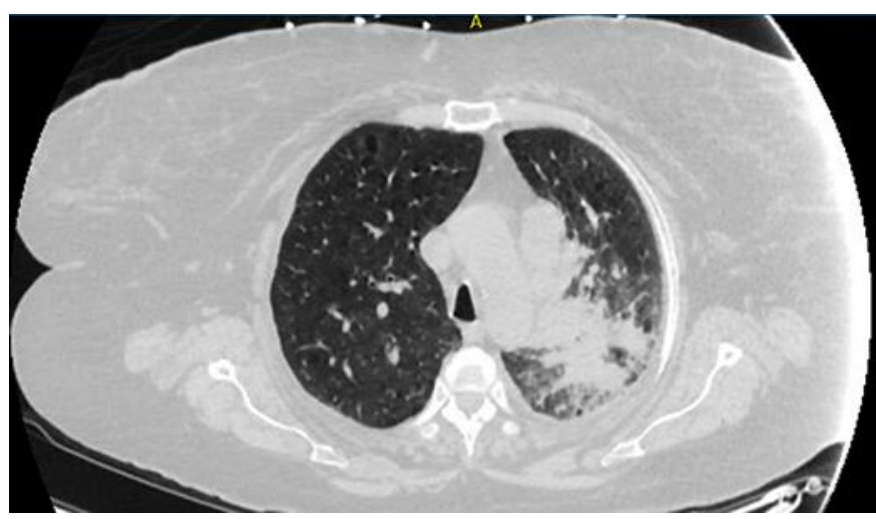

Fig. 1. Chest computed tomography image showing a left upper lung mass associated with obstructive pneumonitis involving much of the left upper lobe. 